\title{
Complexity and network dynamics in physiological adaptation: An integrated view
}

\section{Citation}

Baffy, György, and Joseph Loscalzo. 2014. "Complexity and Network Dynamics in Physiological Adaptation: An Integrated View." Physiology \& Behavior 131 (May): 49-56. doi:10.1016/ j.physbeh.2014.04.018.

\section{Published Version}

doi:10.1016/j.physbeh.2014.04.018

\section{Permanent link}

http://nrs.harvard.edu/urn-3:HUL.InstRepos:34864845

\section{Terms of Use}

This article was downloaded from Harvard University's DASH repository, and is made available under the terms and conditions applicable to Other Posted Material, as set forth at http:// nrs.harvard.edu/urn-3:HUL.InstRepos:dash.current.terms-of-use\#LAA

\section{Share Your Story}

The Harvard community has made this article openly available.

Please share how this access benefits you. Submit a story.

\section{Accessibility}


Complexity and network dynamics in physiological adaptation: An integrated view

György Baffy, MD, $\mathrm{PhD}^{1,2}$ and Joseph Loscalzo, MD, $\mathrm{PhD}^{2}$

Affiliations: Departments of Medicine, ${ }^{1}$ VA Boston Healthcare System and ${ }^{2}$ Brigham and Women's Hospital, Harvard Medical School, Boston, MA

Correspondence: Dr. György Baffy, VA Boston Healthcare System, 150 S. Huntington Ave., Room 6A-46, Boston, Massachusetts 02130. Email: gbaffy@partners.org. Phone: 1-857-364-4327. Fax: 1-857-364-4179.

Word count: 151 (Abstract); 4,662 (Main text). References: 104; Tables: 1; Figures: 3 


\section{Abstract}

Living organisms constantly interact with their surroundings and sustain internal stability against perturbations. This dynamic process follows three fundamental strategies (restore, explore, and abandon) articulated in historical concepts of physiological adaptation such as homeostasis, allostasis, and the general adaptation syndrome. These strategies correspond to elementary forms of behavior (ordered, chaotic, and static) in complex adaptive systems and invite a network-based analysis of the operational characteristics, allowing us to propose an integrated framework of physiological adaptation from a complex network perspective. Applicability of this concept is illustrated by analyzing molecular and cellular mechanisms of adaptation in response to the pervasive challenge of obesity, a chronic condition resulting from sustained nutrient excess that prompts chaotic exploration for system stability associated with tradeoffs and a risk of adverse outcomes such as diabetes, cardiovascular disease, and cancer. Deconstruction of this complexity holds the promise of gaining novel insights into physiological adaptation in health and disease.

Keywords: homeostasis, allostasis, complex adaptive systems, chaotic exploration, phase space, attractor networks 


\section{Introduction}

Living organisms safeguard their integrity and survival against internal and external challenges through adaptation. This dynamic process involves monitoring deviations from the norm, developing appropriate responses to correct the impact of perturbations, and verifying outcomes by sampling internal parameters [1-4]. Adaptation has been extensively studied at successive organizational levels of the biosphere, ranging from unicellular organisms to human society [5-7]. Adaptation may occur at different time scales. Evolutionary adaptation pertains to changes in heritable (i.e., genetic and epigenetic) components of a species or population that accumulate and transfer over many generations [8, 9], while physiological adaptation describes how individual organisms, enabled (and limited) to do so by their unique genome, respond to a variety of day-to-day challenges within a lifetime $[3,4]$. Thus, the primary goal of evolutionary adaptation is to maintain reproductive fitness, while physiological adaptation is more concerned with maintaining energy efficiency and finding the best possible answer to lifetime encounters. Nevertheless, evolutionary and physiological adaptations share many features and complement each other as natural selection acts through individuals.

Since the pioneering work of Claude Bernard [10], our understanding of adaptation in biological systems has benefited from a large body of observational and experimental work. Physiologists, neuroendocrinologists, and behavioral and social scientists developed new theories to interpret the ways by which adaptation may take place in individual organisms. However, a century and a half after Bernard introduced his groundbreaking concepts, there is still an ongoing dispute about the framework of physiological adaptation and how to best apply this knowledge in medicine that primarily aims to prevent and cure dysfunction of this process manifesting as disease [11-15]. 
Parallel with these efforts, basic operational principles of life as a dissipative system have been defined by mathematical and thermodynamic reasoning [16, 17]. More recently, complex network science has provided new tools to study human physiology and offered new opportunities on disease definition, outcomes prediction, and personalized therapy $[18,19]$. Here we aim to integrate the advances in three major fields of biomedical research to define a comprehensive framework of physiological adaptation. First, we review parallel and competing concepts of physiological adaptation providing the fundamental principles of regulating the integrity of living systems. Second, we evaluate these theories in the context of life as a complex adaptive system. Third, we analyze physiological adaptation from a network perspective to describe common structural elements, operational patterns, and regulatory circuitries. Throughout the paper, we use obesity as a prototype of chronic complex diseases to demonstrate the relevance and utility of this integrative approach.

\section{Historical concepts of physiological adaptation}

\subsection{Homeostasis}

Claude Bernard was the first to recognize the importance of constant dialogue between organism and environment [10]. Bernard's key legacy is the notion of milieu intérieur or internal environment, which is in a dynamic equilibrium that must be preserved in all living systems [10]. Based on these principles, Cannon introduced the term homeostasis as the ability to sustain various physiological parameters in a steady state (stabilized around a set point) amidst wide fluctuations in external conditions [20]. Although Cannon illustrated homeostasis by relatively simple examples, such as regulation of thirst and acid-base balance, he intended to use the concept in a broader sense [5]. Indeed, the paradigm of homeostasis contains many conceptual elements on which we may continue to improve our view of physiological integrity and development of disease [12]. 


\subsection{Stress and the general adaptation syndrome}

An interesting aspect of physiological adaptation was highlighted by the work of Selye, who found that experimental animals responded with surprisingly similar clinical symptoms to a wide range of acute physical, chemical, biological, or psychological stimuli, and this response uniformly included activation of the hypothalamic-pituitaryadrenal axis [21, 22]. Selye chose the term 'stress' for this consistent reaction and 'stressors' for the noxious stimuli. He subsequently observed that patients with different diseases exhibited common symptoms of stress and introduced the concept of general adaptation syndrome [23]. In his concept, Selye distinguished the stages of alarm, resistance, and exhaustion to describe how prolonged stress becomes a major challenge to homeostasis with failing adaptation that may culminate in all-consuming disease [23]. The ability of each individual to tailor the magnitude and outcome of this generic response to stress, however, remained difficult to predict.

\subsection{Complementary models of physiological adaptation}

The following decades have seen an expansion of interest in the physiology of adaptation and resulted in parallel growth of related concepts. This trend highlighted some important aspects that were not fully elaborated within the original homeostasis theory [12]. Waddington used the term homeorhesis to describe the goal of physiological control as a trajectory rather than a set point [24]. Selye added heterostasis to distinguish adaptation that reaches a new equilibrium from one that reestablishes original physiological parameters [25]. Moore-Ede proposed a distinction between reactive and predictive homeostasis to emphasize the difference between adaptive strategies that occur in the wake of perturbations as opposed to those initiated in anticipation of predictable changes [26]. 


\subsection{Allostasis and allostatic load}

The concept of allostasis was proposed in 1988 as a comprehensive effort to address some of the perceived limitations of homeostasis [27]. Allostasis is defined as a way to maintain stability through changes by adapting to both predictable and unpredictable events [28]. Key elements of allostasis include shifting set points, alternative pathways, and coordination across multiple regulatory systems orchestrated by the brain $[29,30]$. Allostasis has proven to be a useful framework to assess the impact of neurobehavioral and psychosocial factors in areas where anticipatory physiological regulation is essential such as developmental changes, reproductive cycles, diurnal variations, and in adverse situations such as addiction and post-traumatic stress disorder [30-32].

While the utility of allostasis as yet another term of physiological adaptation remains debated [11, 12, 14], allostatic load has been introduced to address long-term consequences of adaptation, combining relevant elements of homeostasis and chronic stress [33]. Allostatic load refers to the aggregate impact of physiological adaptation over the lifespan of an individual, corresponding to a summary effect of tradeoffs, compromises, and collateral damage [33]. Allostatic load may accumulate faster than expected if sustained activation of regulatory networks exceeds optimal operating ranges either because of excessive duration, frequency, or intensity of perturbations [34]. This accelerated process has been designated as allostatic overload and proposed to correlate with increased vulnerability and risk for development of disease [28]. However, prediction of an individual's ability to limit the accumulation of allostatic load and identification of the specific components that do so remain difficult.

\subsection{Inflammation as an adaptation response}


An intriguing concept was recently introduced by Okin and Medzhitov to redefine the role of inflammation within the context of physiological adaptation [35]. Accordingly, full-scale biological performance in specialized tissues depends on accessory cells originating in the immune system. Due to their mobility and ability to connect various tissues, these accessory cells are the 'common currency' that brings similar operational principles to these sites [35]. Normally, resident 'client' cells successfully cope with perturbations to maintain homeostasis. If this level of physiological adaptation becomes insufficient, accessory cells are recruited to initiate inflammation for a heightened level of tissue adaptation that dominates over homeostasis and involves collateral damage [35]. In this sense, inflammation can be defined as a mechanism of allostasis.

Inflammation is particularly relevant to obesity and its associated adverse health conditions, such as type 2 diabetes, cardiovascular disease, and cancer [36-38]. Aimed at accommodating sustained nutrient excess, adipose tissue growth and remodeling is central to the pathogenesis of obesity, featuring macrophage infiltration and secretion of pro-inflammatory adipokines such as leptin, resistin, tumor necrosis factor [TNF]-alpha, and interleukin [IL]-6 [36]. The ensuing systemic low-grade inflammation promotes a multitude of pathological and self-perpetuating events, such as insulin resistance, endothelial dysfunction, and activation of oncogenic pathways [36, 39]. As discussed further below, obesity is an important example of how environmental factors can create unprecedented challenges for physiological adaptation.

\subsection{Strategies of physiological adaptation in historical concepts}

Three distinct strategies of physiological adaptation can be inferred from the concepts discussed above. First, when the status quo is indispensable or remains preferable over change, biological systems restore current parameters to values that preceded the 
perturbation. This is the dominant strategy of homeostasis. Second, biological systems may explore alternative states to find a new balance (set point) with the environment, in particular if the perturbation has excessive duration, frequency, or intensity. There may be anticipatory elements in this process as living organisms tend to track variations or even alter the environment (e.g., niche construction) to secure physiological integrity and survival [4]. In many situations, this may be a preferable strategy (e.g., inflammation) even if there are compromises and collateral damage involved, as outlined in the concepts of general adaptation syndrome and allostasis. Third, physiological adaptation may require the organism to abandon some of its functions or components (e.g., removing a group of cells by apoptosis) and avoid the spread of system disruption, which may ultimately result in death. Essentially all concepts related to physiological integrity are based on one or more of these distinct adaptation strategies (Table 1).

\section{Chaos and complexity in physiological adaptation}

Biological systems acquire free energy and substances from the environment that are subsequently returned in a degraded form. This exchange allows living organisms to adapt to the surrounding world and protect their inner order despite an endless string of disruptive events (i.e., chronic stress) [34, 40]. Accordingly, the primary role of physiological adaptation is to find the energetically most efficient system configuration that may best guarantee the survival of an individual in a given situation. In this sense, aging and disease indicate a system-wide deterioration with declining efficiency of energy capture and utilization in biological systems until they invariably fail and succumb to death [41].

There is now evidence that basic operational rules of living systems follow some relatively simple mathematical principles, which apply to all levels of the biosphere, 
ranging from genetic blueprints to protein-protein interactions to social networks [42, 43]. Life reflects all the key characteristics of complex systems: living organisms have a large number of interdependent constituents that often behave chaotically, span across several organizational scales, show collectivity and emergence, and maintain a balance between cooperation and competition [44]. As discussed below, each of these attributes has profound implications for physiological adaptation.

\subsection{Chaos and entropy}

Chaos theory provides a framework for the analysis of nonlinear changes in space and time. Chaos in space refers to a structure that does not become simpler when analyzed with deeper and deeper resolution (e.g., fractals of a rugged shoreline). Space-chaos renders reductionist analysis of biological systems a virtually endless quest. Chaos in time refers to the trajectory of a changing system that cannot be accurately predicted. Time-chaos results from sensitivity to initial conditions, indicating that even the smallest uncertainties in a changing system may grow exponentially with time and make current knowledge obsolete [44]. Notable examples of this behavior include the weather and the economy. Since dynamical systems may take alternative paths at every step of the change, chaotic behavior occurs even if each action follows predetermined rules. Chaotic features of living systems can be illustrated by the difficult task of predicting disease outcome in an individual or tracking the whereabouts of a clever fugitive.

Any given state of a dynamical system can be assigned to a single point in a multidimensional phase space, which includes all possible conditions. With time, dynamical systems move from one point to another, and a growing area of probability distribution will represent the cumulative effect of choices between multiple possible states (Figure 1). Following the examples above, meteorologists apply this principle when they forecast 
the trajectory of a hurricane by mapping an ever-widening swath to mark its future position, while stock market predictions are based on a similar process. We have also witnessed growing (rather than shrinking) lists of differential diagnoses and extended search maps to find runaway renegades.

With the lapse of time, the configuration of a dynamic system may assume any point in the phase space, eventually making prediction a useless exercise. Uniform distribution carries minimal information and maximized entropy, which in this case can be mathematically defined as both lack of order and lack of knowledge [44]. Entropy in chaotically behaving systems is proportional to the number of choices that exponentially accumulate through successive steps [44]. In complex adaptive systems, chaotic exploration is geared toward finding the energetically most efficient state [45]. Thus, perturbations prompt a chaotic search in the phase space for alternative solutions from which the system may benefit, and this is exactly the goal of physiological adaptation.

\subsection{Structure, emergence, and self-organization}

The biosphere consists of hierarchical scales with constituents, such as elementary particles, molecules, cells, tissues, organs, individuals, and ecosystems, which interact and acquire specialized functions. These complex structures show emergence, indicating that knowing the individual components is insufficient to understand the result of their collective behavior [42]. For instance, inflammation, consciousness, or legislation cannot be simply deciphered from even the most detailed knowledge of individual macrophages, brain cells, or politicians respectively. Through an iterative process of chaotic search, perturbations may prompt system constituents to interact globally and find an emergent response, which is more than the sum of its parts and becomes regulated at a higher scale. This mechanism is the basis of self-organization [46]. 
Self-organization indicates an ability to create order from disorder without the need of an external organizing force. This ability is fundamental to living organisms that constantly remain in non-equilibrium and aim to keep inner entropy at low levels [46]. In complex adaptive systems, spontaneous emergence of an alternative order may provide the best response to a new situation. Environmental fluctuations encouraging phase space exploration are critical to the process of adaptation. For instance, increasingly rich environments (e.g., through niche construction) enhance behavioral learning and evolutionary complexity [47]. Thus, structure and function can improve by going through cycles of adaptation that involve chaotic search and uncertainty before reaching a new level of self-organization. In this sense, self-organization complements natural selection in the evolutionary process [48-50].

\subsection{Cooperation and competition}

Cooperation is working together for a common purpose, while competition is seeking control over the same or overlapping resources. Cooperation is associated with actions, such as communication, synergy, recognition, tolerance, and altruism, while competition is associated with isolation, antagonism, rejection, intolerance, and selfishness [51, 52]. Yet, competition has important benefits by leading to innovative and more efficient solutions. These opposing forms of behavior coexist in complex systems where components often compete on one scale $(n)$ and cooperate on the coarser scale $(n-1)$ above it. Competition is often intransitive (e.g., A fights B and B fights C, but A does not fight C) and localized in physical space (segregated), which may make overall system behavior more complex and chaotic [52]. Competition and cooperation may also occur within one organizational scale when competing constituents employ coordinated rather than individual effort to reach their goal (e.g., gang-like behavior). 


\subsection{Strategies of physiological adaptation in complex systems}

Motion of a single point or trajectory, representing an elementary functional unit of a biological system in phase space, may follow ordered dynamics and exhibit change in a linear (predictable) way and proportional to the perturbations. In many cases, this controlled change is transient and serves restoration of a critical parameter to its original value (Figure 1A). Subsets of the phase space in which a dynamic system may find stability and tends to return over time are called attractors [53]. Returning to the original attractor or set point is the preferred response of homeostasis as originally defined by Cannon [20]. In other cases, perturbed elementary functional units may find alternative attractors by following nonlinear dynamics through chaotic exploration (Figure 1B). This response is unpredictable and could be disproportionate, a scenario aptly described by the allostasis theory [54]. Finally, biological systems may fail to respond (e.g., stressinduced exhaustion, allostatic crash, or death), indicating a static condition (Figure 1C).

These operational principles can be readily applied to analyze mechanisms of adaptation to regulate energy metabolism in obesity. Perturbation analysis in human erythrocytes based on structural kinetic modeling indicates that system stability is increasingly sensitive to changing fluxes in ATP production or consumption [55]. In addition, fuzzy-logic simulation and sensitivity analyses indicate that manipulating ATP regulatory components makes metabolic regulation highly sensitive to smaller perturbations [56]. Persistent and substantial surplus or shortage in energy substrates of the cell may generate stress in the metabolic phase space that initiates a chaotic search for alternative attractors. Thus, homeostatic regulation of energy metabolism in obesity may give way to allostatic mechanisms, such as inflammation [35] and altered energy metabolism, as discussed below. Chaotic exploration for system stability initiated by 
persistent caloric surplus may then find increasingly unusual attractors linked to the development of obesity-associated adverse health events, such as diabetes and cancer.

\section{Network properties of physiological adaptation}

Network analysis has become a powerful tool of systems biology efforts to deconstruct the complexity of living organisms. Networks (graphs) consist of nodes (vertices) connected with links (edges) through some kinds of architecture (topology). Possible interactions between groups of nodes (subgraphs) can be described mathematically, modeled by mechanical circuitries, and compared to real-life presence in biological systems [57, 58]. What can networks tell us about homeostasis? What network topology is associated with ordered and chaotic adaptive responses? Can we identify network attributes that help or hinder our ability to cope with stress and prevent disease? Are there changes in network behavior that may predict the success or failure of this process? Finding answers to these questions may offer new perspectives in the understanding of physiological adaptation.

Based on their topology, networks may follow different principles of organization. In random networks, connections among nodes are driven by chance, resulting in a Poisson distribution for the numbers of links per node [59]. In scale-free networks, the distribution of links per node follows a power law, indicating that most nodes have few connections while a small number of nodes have a progressively high number of links [60]. Preferential attachment ('the rich get richer') is a basic property of evolving scalefree networks, with new nodes more likely to be added to one of the highly connected nodes (hubs) rather than to a wealthy connected node [60]. Since its original formulation a little over a decade ago [60], the theory of scale-free networks has become a pervasive platform for studying complex systems. Scale-free networks have surprisingly 
uniform architectural features and rules of operations, regardless of being abstract, inanimate, or alive [57] (Figure 2).

\subsection{Feedback and other network motifs}

Network dynamics depend on the types of interactions between nodes and links. Although the theoretical number of these interactions can be very large, there are surprisingly few such patterns of interaction (network motifs) observed in biological systems (e.g., sensory transcription, signal transduction, developmental, or neuronal networks) [61]. A feedback loop is an elementary form of signal control and one of the best-known network motifs associated with physiological adaptation. Feedback has a corrective effect on the input by monitoring system status, stabilizing responses to perturbations [62]. As indicated by mathematical modeling, feedback is essential for allosteric regulation and greatly improves the dynamic properties and stability of metabolic networks [55]. This stabilizing effect of feedback control protects the system against widely varying external conditions, indicating a key role for allosteric enzymes in maintaining and restoring metabolic homeostasis [55].

While negative feedback diminishes the impact of perturbation, positive feedback acts as a signal amplifier at the expense of unpredictable responses (autocatalysis) [63]. A special form of positive feedback, which involves a heterodimer where only one component is subject to feedback regulation, is designed to limit the risk of runaway mechanisms. This circuit is based on an asymmetric self-upregulation (ASSURE) motif that allows a strong yet precisely tuned response in the presence of varying signal intensity [64]. ASSURE is utilized in many biological systems, such as cellular antiviral defense, cholesterol homeostasis, and adipocyte differentiation [64]. 
Mitochondrial respiration is an important example of positive feedback in metabolic networks. While oxidative phosphorylation is a highly efficient mechanism of ATP synthesis, it is also a key site of superoxide production, making mitochondria the primary source of oxidative stress in the cell [65]. Superoxide forms when electrons spin off the respiratory chain before reaching cytochrome oxidase and react with molecular oxygen. This process may be amplified if electron flow becomes sluggish due to oxidative damage of respiratory complexes, creating a vicious cycle [66]. Importantly, increased supply of respiratory substrates (e.g., due to increased caloric intake in obesity) promotes early spinoff of electrons by slowing their flow against the high proton gradient [67]. Uncoupling proteins, activated by superoxide, may prevent this electron pile-up by deflating the proton gradient, but diminished ATP synthesis remains a substantial tradeoff [68].

Regulation of oxidative phosphorylation features additional feedback circuits serving the prevention of excessive ROS production while shifting ATP production away from the mitochondria. Thus, decreased iron-sulfur protein assembly in the electron transport chain mediated by microRNA-210 and dampened flux of the tricarboxylic acid cycle mediated by pyruvate dehydrogenase kinase 1 in response to hypoxia are under the control of hypoxia-inducible factor HIF-1, both mechanisms aimed at reducing mitochondrial oxidative stress while promoting ATP synthesis via glycolysis $[69,70]$. This fundamental mechanism of adaptation, known as the Pasteur effect, conceals a major tradeoff (i.e., the Warburg effect) that allows metabolic reprogramming in rapidly proliferating cancer cells [71].

\subsection{Redundancy and degeneracy}


Redundancy in a network indicates having more than one node or clusters of nodes to perform identical or similar functions, resulting in the multiplication of critical components or functions [72]. Redundancy is the mirror image of multi-functionality (bow-tie architecture) with a single node involved in more than one pathway. In structural redundancy, multiple copies of a given element enhance system reliability. Gene duplication is a classical example of this type of redundancy in biological systems. Provision of more than one means or resource to perform the same action is functional redundancy [72]. Biological systems often engage different structures for realizing same or very similar outcomes. This property, also called degeneracy, can be found between various developmental pathways or signaling mechanisms that are redundant only within particular contexts [72]. A classical example for degeneracy is the genetic code in which multiple nucleotide triplets (codons) call for using the same amino acid during protein synthesis.

An important difference between redundancy and degeneracy is that degenerate systems are able to produce different outputs in different contexts, which makes them extremely adaptive [72]. Sustained and high-level degeneracy allows the use of alternative pathways in response to unpredictable perturbations (e.g., single point failure). A network with little functional redundancy is brittle but responds in predictable ways, while a redundant network may use different outputs to yield equivalent functions [73]. This is an important tradeoff between ordered and chaotic responses, and may be utilized to characterize adaptive responses under increasing environmental pressure.

Adipose tissue remodeling associated with secretion of a multitude of pro-inflammatory cytokines, chemokines, adhesion molecules, and adipokines exemplifies redundancy associated with obesity [74]. These biologically active substances promote insulin 
resistance, contribute to endothelial dysfunction, and activate oncogenic pathways by exerting parallel effects on downstream signaling cascades and gene expression [75-77]. The resultant structural and functional redundancy may substantially limit success in finding molecular targets at this operational level to prevent the adverse effects of obesity.

\subsection{Modularity and network diameter}

Modules in scale-free networks represent areas with highly interconnected nodes involved in similar or sequentially arranged functions (e.g., metabolic pathways or signaling cascades) [78]. Network modules are characterized by high clustering coefficients (defined as the ratio of existing over possibly existing links), indicating that any two neighbors of a given node are likely linked to each other [57]. Links within a module are short and strong, while different modules are connected by longer and weaker links. Highly modular networks are stable against random perturbations, and allow the on-off switch of individual modules without affecting other functions (pleiotropy) $[78,79]$. These beneficial properties depend on the degree of component sharing (overlap) between different modules.

Network diameter is defined as the greatest distance between any pair of nodes by selecting the shortest possible route. There is a tradeoff between adaptive speed and accuracy as responses modeled in small gene networks are rapid but inaccurate, while large networks have accurate responses that may take a longer time [80]. In addition, network diameters increase with the extent of modularity in mathematical simulations, indicating another tradeoff between efficiency and pleiotropy [81]. For instance, metabolic flux and modularity analysis of 3T3-L1 pre-adipocytes indicates that major reaction clusters of lipid metabolism coalesce into a single module by day 8 in culture 
with a primary biochemical function to convert glucose into triglycerides [82]. These observations suggest that de novo adipocyte formation, an important element of higher adipocyte turnover in obesity [83], is associated with an increasingly centralized metabolic network in which preference is given to response velocity (which is heightened by increased clustering) over pleiotropy.

\subsection{Robustness and fragility}

Complex systems with stable characteristics and specific functions amidst changing conditions are described as robust [84]. Network robustness indicates resilience to removal of nodes or links (topological robustness) [85]. Loss of link capacity (weight) or directionality (information flow or direction of the dependencies) in adapting networks may also impair abilities to address properly internal or external challenges [85]. Scalefree networks have a remarkable tolerance for disintegration of their architecture with preserved functionality even if $80 \%$ of nodes are removed by random selection [86]. However, selective removal of highly connected nodes (hubs) in scale-free networks may result in rapid collapse indicating that these systems are robust yet fragile in response to targeted attacks [86].

Robustness is a key attribute of homeostasis, with the original function preserved during adaptation following reliable and ordered pathways. Robustness may also succeed when outcomes are less predictable and adaptive responses have elements of chaotic exploration. Obesity is a case in point for how energy metabolism, designed to prevent the impact of malnutrition, attains a new (albeit dysfunctional) level of stability in the era of sustained nutrient excess [74]. To maintain normoglycemia under fasting conditions, TNF-alpha blocks peripheral glucose uptake by promoting insulin resistance in skeletal muscle and adipose tissue (Fernandez-Real and Ricart, 1999), while UCP2 blocks 
insulin secretion via interference with ATP-dependent glucose sensing in beta cells [87, 88]. These compensatory mechanisms backfire in food abundance when insulin resistance is robustly maintained by an emergent positive feedback loop involving TNFalpha derived from remodeled adipose tissue [74]. Similarly, persistent abundance of UCP2 in obesity causes dysfunction of the pancreatic beta cell, contributing to endorgan damage [89].

\subsection{Network rewiring and disease}

Node and edge failure is a common cause of deteriorating network performance, while addition of new nodes or links (random or preferential rewiring) to existing networks may increase robustness [90]. Increasing the number of weak ties in simulated and real networks (e.g., peer-to-peer social groups of the Internet) allows faster and more efficient adaptation $[81,90]$. Rewiring has a profound impact on biological networks where topology may be altered by aging or disease. A classic example of molecular rewiring is the $B C R-A B L$ fusion oncoprotein, which results from the Philadelphia chromosome translocation $\mathrm{t}(9 ; 22)(\mathrm{q} 34 ; \mathrm{q} 11)$ and becomes a defining event in chronic myelogenous leukemia (CML) [91]. In CML, BCR-ABL uncouples the JAK-STAT signaling module and allows direct nuclear translocation of STAT5 with enhanced transcription of genes involved in cell growth and differentiation [92].

Replacement of missing genes (e.g., stem cell transplant to replace the $A B C D 1$ gene in adrenoleukodystrophy) or macromolecules (e.g., clotting factors in hemophilia) represents successful attempts at rewiring biological networks [93, 94]. Genetic engineering of supplemental gut bacteria that are traditionally considered as 'probiotics' is another exciting application of therapeutic rewiring. For instance, commensal strains of Escherichia coli transfected to express insulinotropic proteins GLP-1 and PDX-1 
stimulate intestinal epithelial cells to secrete insulin in response to glucose as an effort to treat diabetes [95].

Rewiring the regulation of human energy metabolism in the central nervous system is a particularly intriguing approach to study the pathogenesis of obesity, as correcting faulty neuronal circuits in the hypothalamus may restore homeostatic regulation of appetite and body weight [96]. Thus, administration of leptin may control appetite and other complex behaviors by synaptic reorganization between excitatory and inhibitory contacts on neuropeptide $\mathrm{Y}$ and pro-opiomelanocortin (POMC) neurons [97]. Similar synaptic plasticity can be achieved by using estradiol to increase the number of excitatory inputs to POMC neurons in the arcuate nucleus with a corresponding decrease in appetite and adiposity in leptin-deficient $o b / o b$ mice [98].

\section{Conclusions}

Living organisms constantly interact with their surroundings and regulate this dialogue by matching inherited and acquired abilities to specific challenges through physiological adaptation. In response to various perturbations, biological systems may restore, explore, or abandon ways and means to enhance their development and safeguard their existence. From a systems-level approach, these fundamental adaptation strategies fit the conceptual framework of chaos and complexity surprisingly well. In addition, the complex interplay between constituents of biological systems can be described and predicted by network-based analysis, which provides new perspectives on the understanding of health and disease. Thus, integration of classic physiology, chaos and complexity theory, and network science offers a fresh look at physiological adaptation. 
This conceptual platform is helpful to examine the role of physiological adaptation in complex diseases. Outcomes in these conditions depend on adaptive interactions between a multitude of genetic and environmental factors occurring over a lifetime. Disease progression indicates increasing dysfunction from health to pathophysiological conditions, intermediate phenotypes, and clinically obvious disease pathophenotypes [18]. This process can be displayed within a phase space diagram as a widening probability distribution, indicating a shift from predictable and often transient changes to an escalating search for appropriate responses within varying conditions that threaten the integrity of an individual (Figure 3A). Obesity as a complex disease with widely different outcomes can serve as an excellent model for studying progressively nonlinear trajectories of physiological adaptation (Figure 3B).

Network-based analysis of complex diseases offers additional considerations. Recent work indicates that attractor landscape analysis of vascular, metabolic, and oncogenic pathophenotypes is a useful approach to understand disease pathogenesis and identify novel therapeutic strategies [99-102]. Drawing analogy from the evolutionary concept of adaptive landscapes [103], attractors as alternative states of system stability explored by physiological adaptation in the multidimensional phase space can be viewed as a distinct network (Figure 3C). In principle, such attractor networks may be designed for all genotype-phenotype interactions in a given individual and analyzed by network-based methods if sufficient information and computational power are available. This approach may better define disorders of physiological adaptation and personalized molecular targets for intervention.

\section{Supporting Information}

This work received no grants or other financial support. 


\section{References}

[1] Bennett, A. F. Adaptation and the evolution of physiological characters. In: Dantzler WH, ed. Handbook of Physiology: Section 13. Comparative Physiology. New York: Oxford University Press; 1997. p. 3-16.

[2] Garland, T., Jr., Carter, P. A. Evolutionary physiology. Annu. Rev. Physiol. 1994,56:579-621.

[3] Raubenheimer, D., Simpson, S. J., Tait, A. H. Match and mismatch: conservation physiology, nutritional ecology and the timescales of biological adaptation. Philos. Trans. R. Soc. Lond. B. Biol. Sci. 2012,367:1628-46.

[4] Whitacre, J. M. Biological robustness: paradigms, mechanisms, and system principles. Front. Genet. 2012,3:1-15.

[5] Cannon, W. B. The Wisdom of the Body. New York: Norton; 1932.

[6] Schweiger, H. G., Schweiger, M. Circadian rhythms in unicellular organisms: an endeavor to explain the molecular mechanism. Int. Rev. Cytol. 1977,51:315-42.

[7] Trojan, P. Ecosystem homeostasis. The Hague ; Boston: Dr. W. Junk Publishers : distributed by Kluwer Boston, Inc. ; Warszawa : PWN-Polish Scientific Publishers; 1984.

[8] Maynard Smith, J. The theory of evolution. 3rd ed. ed. Cambridge: Cambridge University Press; 1993.

[9] Fusco, G., Minelli, A. Phenotypic plasticity in development and evolution: facts and concepts. Introduction. Philos. Trans. R. Soc. Lond. B. Biol. Sci. 2010,365:547-56.

[10] Bernard, C. Leçons sur les propriétés physiologiques et les altérations pathologiques des liquides de l'organisme. Paris: Baillière; 1859. 
[11] Dallman, M. F. Stress by any other name .....? Horm. Behav. 2003,43:18-20; discussion 8-30.

[12] Carpenter, R. H. Homeostasis: a plea for a unified approach. Adv Physiol Educ. 2004,28:180-7.

[13] Day, T. A. Defining stress as a prelude to mapping its neurocircuitry: no help from allostasis. Prog. Neuropsychopharmacol. Biol. Psychiatry. 2005,29:1195200.

[14] McEwen, B. S., Wingfield, J. C. What is in a name? Integrating homeostasis, allostasis and stress. Horm. Behav. 2010,57:105-11.

[15] Romero, L. M., Dickens, M. J., Cyr, N. E. The Reactive Scope Model - a new model integrating homeostasis, allostasis, and stress. Horm. Behav. 2009,55:375-89.

[16] Schrödinger, E. What is life? The physical aspect of the living cell. Cambridge Eng.: The University press; 1945.

[17] Nicolis, G., Prigogine, I. Self-organization in nonequilibrium systems : from dissipative structures to order through fluctuations. New York ; London: Wiley; 1977.

[18] Loscalzo, J., Kohane, I., Barabási, A. L. Human disease classification in the postgenomic era: a complex systems approach to human pathobiology. Mol Syst Biol. 2007,3:124.

[19] Barabási, A. L., Gulbahce, N., Loscalzo, J. Network medicine: a network-based approach to human disease. Nat Rev Genet. 2010,12:56-68.

[20] Cannon, W. B. Organization for physiological homeostasis. Physiol. Rev. 1929,9:399-431.

[21] Selye, H. Adaptation energy. Nature. 1938,141:926. 
[22] Selye, H. The general adaptation syndrome and the diseases of adaptation. J. Clin. Endocrinol. Metab. 1946,6:117-230.

[23] Selye, H. Stress and the general adaptation syndrome. Br. Med. J. 1950,1:138392.

[24] Waddington, C. H. The strategy of the genes. London, United Kingdom: George Allen \& Unwin; 1957.

[25] Selye, H. Homeostasis and heterostasis. Perspect. Biol. Med. 1973,16:441-5.

[26] Moore-Ede, M. C. Physiology of the circadian timing system: predictive versus reactive homeostasis. Am. J. Physiol. 1986,250:R737-52.

[27] Sterling, P., Eyer, J. Allostasis: a new paradigm to explain arousal pathology. In: Fisher S, Reason J, eds. Handbook of life stress, cognition, and health. New York1988.

[28] McEwen, B. S., Wingfield, J. C. The concept of allostasis in biology and biomedicine. Horm. Behav. 2003,43:2-15.

[29] Sterling, P. Allostasis: A model of predictive regulation. Physiol. Behav. 2012,106:5-15.

[30] Power, M. L., Schulkin, J. Maternal obesity, metabolic disease, and allostatic load. Physiol. Behav. 2012,106:22-8.

[31] Seeman, T., Epel, E., Gruenewald, T., Karlamangla, A., McEwen, B. S. Socioeconomic differentials in peripheral biology: cumulative allostatic load. Ann. N. Y. Acad. Sci. 2010,1186:223-39.

[32] George, O., Le Moal, M., Koob, G. F. Allostasis and addiction: Role of the dopamine and corticotropin-releasing factor systems. Physiol. Behav. 2011,106:58-64.

[33] McEwen, B. S., Stellar, E. Stress and the individual. Mechanisms leading to disease. Arch. Intern. Med. 1993,153:2093-101. 
[34] Schulkin, J. Allostasis, Homeostasis, and the Costs of Adaptation. Oxford: Cambridge University Press; 2004.

[35] Okin, D., Medzhitov, R. Evolution of inflammatory diseases. Curr. Biol. 2012,22:R733-40.

[36] Gregor, M. F., Hotamisligil, G. S. Inflammatory mechanisms in obesity. Annu. Rev. Immunol. 2011,29:415-45.

[37] Zalesin, K. C., Franklin, B. A., Miller, W. M., Peterson, E. D., McCullough, P. A. Impact of obesity on cardiovascular disease. Med. Clin. North Am. 2011,95:91937.

[38] Calle, E. E., Rodriguez, C., Walker-Thurmond, K., Thun, M. J. Overweight, obesity, and mortality from cancer in a prospectively studied cohort of U.S. adults. N. Engl. J. Med. 2003,348:1625-38.

[39] Louie, S. M., Roberts, L. S., Nomura, D. K. Mechanisms linking obesity and cancer. Biochim. Biophys. Acta. 2013.

[40] Selye, H. The Stress of Life. New York: McGraw-Hill; 1956.

[41] Soltow, Q. A., Jones, D. P., Promislow, D. E. A network perspective on metabolism and aging. Integr Comp Biol. 2010,50:844-54.

[42] Bar-Yam, Y. Dynamics of complex systems. Reading, Massachusetts: AddisonWesley; 1997.

[43] Barabási, A. L. Scale-free networks: a decade and beyond. Science. 2009,325:412-3.

[44] Baranger, M. Chaos, complexity, and entropy : a physics talk for non-physicists. New England Complex Systems Institute: Cambridge; 2000.

[45] Komarov, M. A., Osipov, G. V., Burtsev, M. S. Adaptive functional systems: learning with chaos. Chaos. 2010,20:045119. 
[46] Prigogine, I., Stengers, I. Order out of chaos : man's new dialogue with nature. London: Heinemann; 1984.

[47] Krakauer, D. C. Darwinian demons, evolutionary complexity, and information maximization. Chaos. 2011,21:037110.

[48] Skar, J. Introduction: self-organization as an actual theme. Philos Transact A Math Phys Eng Sci. 2003,361:1049-56.

[49] Kauffman, S. A. Antichaos and adaptation. Sci. Am. 1991,265:78-84.

[50] Hoelzer, G. A., Smith, E., Pepper, J. W. On the logical relationship between natural selection and self-organization. J Evol Biol. 2006,19:1785-94.

[51] Axelrod, R., Hamilton, W. D. The evolution of cooperation. Science. 1981,211:1390-6.

[52] Klimenko, A. Y. Complex competitive systems and competitive thermodynamics. Philos Transact A Math Phys Eng Sci. 2013,371:20120244.

[53] Kauffman, S. A. The origins of order : self organization and selection in evolution. New York: Oxford University Press; 1993.

[54] Sterling, P. Principles of allostasis: optimal design, predictive regulation, pathophysiology and rational therapeutics. In: Schulkin J, ed. Allostasis, Homeostasis, and the Costs of Adaptation. Oxford: Cambridge University Press; 2004.

[55] Grimbs, S., Selbig, J., Bulik, S., Holzhutter, H. G., Steuer, R. The stability and robustness of metabolic states: identifying stabilizing sites in metabolic networks. Mol Syst Biol. 2007,3:146.

[56] Kriete, A., Bosl, W. J., Booker, G. Rule-based cell systems model of aging using feedback loop motifs mediated by stress responses. PLoS Comput Biol. 2010,6:e1000820. 
[57] Albert, R., Barabási, A. L. Statistical mechanics of complex networks. Reviews of Modern Physics. 2002,74:47-97.

[58] Alon, U. An introduction to systems biology : design principles of biological circuits. Boca Raton, FL: Chapman \& Hall/CRC; 2007.

[59] Erdös, P., Rényi, A. On the evolution of random graphs. Publ. Math. Inst. Hung. Acad. Sci. 1960,5:17-61.

[60] Barabási, A. L., Albert, R. Emergence of scaling in random networks. Science. 1999,286:509-12.

[61] Shen-Orr, S. S., Milo, R., Mangan, S., Alon, U. Network motifs in the transcriptional regulation network of Escherichia coli. Nat. Genet. 2002,31:64-8.

[62] Alon, U., Surette, M. G., Barkai, N., Leibler, S. Robustness in bacterial chemotaxis. Nature. 1999,397:168-71.

[63] Levine, W. S. Control system advanced methods. 2nd ed. Boca Raton: CRC Press; 2011.

[64] Ratushny, A. V., Saleem, R. A., Sitko, K., Ramsey, S. A., Aitchison, J. D. Asymmetric positive feedback loops reliably control biological responses. Mol Syst Biol. 2012,8:577.

[65] Turrens, J. F. Superoxide production by the mitochondrial respiratory chain. Biosci. Rep. 1997,17:3-8.

[66] Brand, M. D., Affourtit, C., Esteves, T. C., Green, K., Lambert, A. J., Miwa, S., et al. Mitochondrial superoxide: production, biological effects, and activation of uncoupling proteins. Free Radic. Biol. Med. 2004,37:755-67.

[67] Korshunov, S. S., Skulachev, V. P., Starkov, A. A. High protonic potential actuates a mechanism of production of reactive oxygen species in mitochondria. FEBS Lett. 1997,416:15-8. 
[68] Brand, M. D., Esteves, T. C. Physiological functions of the mitochondrial uncoupling proteins UCP2 and UCP3. Cell Metab. 2005,2:85-93.

[69] Kim, J. W., Tchernyshyov, I., Semenza, G. L., Dang, C. V. HIF-1-mediated expression of pyruvate dehydrogenase kinase: a metabolic switch required for cellular adaptation to hypoxia. Cell Metab. 2006,3:177-85.

[70] Chan, S. Y., Zhang, Y. Y., Hemann, C., Mahoney, C. E., Zweier, J. L., Loscalzo, J. MicroRNA-210 controls mitochondrial metabolism during hypoxia by repressing the iron-sulfur cluster assembly proteins ISCU1/2. Cell Metab. 2009,10:273-84.

[71] Vander Heiden, M. G., Cantley, L. C., Thompson, C. B. Understanding the Warburg effect: the metabolic requirements of cell proliferation. Science. 2009,324:1029-33.

[72] Edelman, G. M., Gally, J. A. Degeneracy and complexity in biological systems. Proc. Natl. Acad. Sci. U. S. A. 2001,98:13763-8.

[73] Tononi, G., Sporns, O., Edelman, G. M. Measures of degeneracy and redundancy in biological networks. Proc. Natl. Acad. Sci. U. S. A. 1999,96:325762.

[74] Kitano, H., Oda, K., Kimura, T., Matsuoka, Y., Csete, M., Doyle, J., et al. Metabolic syndrome and robustness tradeoffs. Diabetes. 2004,53 Suppl 3:S6S15.

[75] Martinez, J. A. Mitochondrial oxidative stress and inflammation: an slalom to obesity and insulin resistance. J. Physiol. Biochem. 2006,62:303-6.

[76] Philip-Couderc, P., Smih, F., Hall, J. E., Pathak, A., Roncalli, J., Harmancey, R., et al. Kinetic analysis of cardiac transcriptome regulation during chronic high-fat diet in dogs. Physiol Genomics. 2004,19:32-40. 
[77] Grant, R. W., Vester Boler, B. M., Ridge, T. K., Graves, T. K., Swanson, K. S. Adipose tissue transcriptome changes during obesity development in female dogs. Physiol Genomics. 2011,43:295-307.

[78] Ravasz, E., Barabási, A. L. Hierarchical organization in complex networks. Phys Rev E Stat Nonlin Soft Matter Phys. 2003,67:026112.

[79] Loscalzo, J., Barabási, A. L. Systems biology and the future of medicine. Wiley Interdiscip Rev Syst Biol Med. 2011,3:619-27.

[80] Malcom, J. W. Evolution of competitive ability: an adaptation speed vs. accuracy tradeoff rooted in gene network size. PLoS One. 2011,6:e14799.

[81] Zhang, Z., Zhang, J. A big world inside small-world networks. PLoS One. 2009,4:e5686.

[82] Si, Y., Yoon, J., Lee, K. Flux profile and modularity analysis of time-dependent metabolic changes of de novo adipocyte formation. Am J Physiol Endocrinol Metab. 2007,292:E1637-46.

[83] Arner, E., Westermark, P. O., Spalding, K. L., Britton, T., Ryden, M., Frisen, J., et al. Adipocyte turnover: relevance to human adipose tissue morphology. Diabetes. 2010,59:105-9.

[84] Bar-Yam, Y., Epstein, I. R. Response of complex networks to stimuli. Proc. Natl. Acad. Sci. U. S. A. 2004,101:4341-5.

[85] Barabási, A. L., Oltvai, Z. N. Network biology: understanding the cell's functional organization. Nat Rev Genet. 2004,5:101-13.

[86] Albert, R., Jeong, H., Barabási, A. L. Error and attack tolerance of complex networks. Nature. 2000,406:378-82.

[87] Fernandez-Real, J. M., Ricart, W. Insulin resistance and inflammation in an evolutionary perspective: the contribution of cytokine genotype/phenotype to thriftiness. Diabetologia. 1999,42:1367-74. 
[88] Chan, C. B., Kashemsant, N. Regulation of insulin secretion by uncoupling protein. Biochem. Soc. Trans. 2006,34:802-5.

[89] Krauss, S., Zhang, C. Y., Scorrano, L., Dalgaard, L. T., St-Pierre, J., Grey, S. T., et al. Superoxide-mediated activation of uncoupling protein 2 causes pancreatic beta cell dysfunction. J. Clin. Invest. 2003,112:1831-42.

[90] Beygelzimer, A., Grinstein, G., Linsker, R., Rish, I. Improving network robustness by edge modification. Physica A. 2005,357:593-612.

[91] Wong, S., Witte, O. N. The BCR-ABL story: bench to bedside and back. Annu. Rev. Immunol. 2004,22:247-306.

[92] Hantschel, O., Warsch, W., Eckelhart, E., Kaupe, I., Grebien, F., Wagner, K. U., et al. BCR-ABL uncouples canonical JAK2-STAT5 signaling in chronic myeloid leukemia. Nat Chem Biol. 2012,8:285-93.

[93] Alexander, I. E., Kok, C., Dane, A. P., Cunningham, S. C. Gene therapy for metabolic disorders: an overview with a focus on urea cycle disorders. J. Inherit. Metab. Dis. 2012,35:641-5.

[94] Cartier, N., Hacein-Bey-Abina, S., Bartholomae, C. C., Veres, G., Schmidt, M., Kutschera, I., et al. Hematopoietic stem cell gene therapy with a lentiviral vector in X-linked adrenoleukodystrophy. Science. 2009,326:818-23.

[95] Duan, F., Curtis, K. L., March, J. C. Secretion of insulinotropic proteins by commensal bacteria: rewiring the gut to treat diabetes. Appl. Environ. Microbiol. 2008,74:7437-8.

[96] Gobel, B., Langemann, D. Systemic investigation of a brain-centered model of the human energy metabolism. Theory Biosci. 2011,130:5-18.

[97] Pinto, S., Roseberry, A. G., Liu, H., Diano, S., Shanabrough, M., Cai, X., et al. Rapid rewiring of arcuate nucleus feeding circuits by leptin. Science. 2004,304:110-5. 
[98] Gao, Q., Mezei, G., Nie, Y., Rao, Y., Choi, C. S., Bechmann, I., et al. Anorectic estrogen mimics leptin's effect on the rewiring of melanocortin cells and Stat3 signaling in obese animals. Nat. Med. 2007,13:89-94.

[99] Wang, W. Therapeutic hints from analyzing the attractor landscape of the p53 regulatory circuit. Sci Signal. 2013,6:pe5.

[100] De la Fuente, I. M., Cortes, J. M., Pelta, D. A., Veguillas, J. Attractor metabolic networks. PLoS One. 2013,8:e58284.

[101] Choi, M., Shi, J., Jung, S. H., Chen, X., Cho, K. H. Attractor landscape analysis reveals feedback loops in the p53 network that control the cellular response to DNA damage. Sci Signal. 2012,5:ra83.

[102] Butcher, J. T., Goodwill, A. G., Stanley, S. C., Frisbee, J. C. Blunted temporal activity of microvascular perfusion heterogeneity in metabolic syndrome: a new attractor for peripheral vascular disease? Am J Physiol Heart Circ Physiol. 2013,304:H547-58.

[103] Wright, S. The roles of mutation, inbreeding, crossbreeding, and selection in evolution. Proc. 6th Int. Congr. Genet. 1932,1:356-66.

[104] Mangum, C., Towle, D. Physiological adaptation to unstable environments. Am. Sci. 1977,65:67-75. 


\section{Tables}

Table 1. Historical concepts and key strategies of physiological adaptation

\begin{tabular}{|c|c|c|}
\hline Restore & Explore & Abandon \\
\hline $\begin{array}{l}\text { Homeostasis: Maintenance of } \\
\text { physiological variables within } \\
\text { optimum range around set } \\
\text { points (steady state) [20] }\end{array}$ & $\begin{array}{l}\text { Heterostasis: Activation of } \\
\text { dormant defensive reactions } \\
\text { to reach new equilibrium in a } \\
\text { changing environment [40] }\end{array}$ & \\
\hline $\begin{array}{l}\text { Homeorhesis: Physiological } \\
\text { stability by making correction } \\
\text { to a trajectory rather than a } \\
\text { single defined set point [24] }\end{array}$ & $\begin{array}{l}\text { Enantiostasis: Replacement of } \\
\text { original regulation by new } \\
\text { mechanisms to maintain equal } \\
\text { or similar functions [104] }\end{array}$ & \\
\hline \multirow[t]{2}{*}{$\begin{array}{l}\text { Alarm: Awareness of danger } \\
\text { (stressor) and activation of } \\
\text { nonspecific defense such as } \\
\text { the HPA axis [23] }\end{array}$} & $\begin{array}{l}\text { Resistance: Reversible } \\
\text { allocation of energy for } \\
\text { continued fight against } \\
\text { persisting stress [23] }\end{array}$ & $\begin{array}{l}\text { Exhaustion: Accumulating } \\
\text { burden of adaptation due to } \\
\text { stress overload, burnout, and } \\
\text { vanishing resistance [23] }\end{array}$ \\
\hline & $\begin{array}{l}\text { Allostasis: Achieving stability } \\
\text { through change, anticipating } \\
\text { need for adjustment, and } \\
\text { abandoning set points for new } \\
\text { ones while accumulating wear } \\
\text { and tear (allostatic load) [ } 27 \text {, } \\
30,33]\end{array}$ & $\begin{array}{l}\text { Allostatic overload: Failing } \\
\text { adaptive regulatory systems } \\
\text { due to sustained activation, } \\
\text { self-perpetuating dysfunction } \\
\text { with increased risk of disease } \\
\text { and death }[33,34]\end{array}$ \\
\hline $\begin{array}{l}\text { Predictive homeostasis: } \\
\text { Corrective response to }\end{array}$ & $\begin{array}{l}\text { Reactive homeostasis: } \\
\text { Response to changes not }\end{array}$ & $\begin{array}{l}\text { Homeostatic overload: } \\
\text { Response to further }\end{array}$ \\
\hline
\end{tabular}




\begin{tabular}{|l|l|l|}
\hline anticipated changes within & anticipated by predictive & challenges resulting in \\
the range of predictable life- & operations, with outcomes still & damage and failure due to \\
history demands [15] & within 'reactive scope' [15] & sustained disruption [15] \\
\hline
\end{tabular}


Figure Legends

Figure 1

\section{Strategies of physiological adaptation}

A. Components of biological systems responding to perturbations exhibit various behaviors in the multidimensional phase space of complex systems. Single point represents a functional unit residing in a small area of probability distribution (red circle) with predictable and reversible positional changes without leaving the original attractor or 'set point' (straight arrows), indicating efficient control of critical parameters by homeostasis. B. Upon more intense or persistent perturbation, units may find stability in new attractors by following nonlinear dynamics through chaotic exploration (curved arrows), which may provide a better match to altered conditions while makes precise position of the unit increasingly unpredictable. This adaptive response, associated with tradeoffs and compromises, is typical for allostasis. C. As perturbation continues, escalating cycles of chaotic exploration may find attractors in which the unit ultimately fails to respond, indicating a static condition and representing maximal entropy. This path is seen in stress-induced exhaustion or allostatic crash representing the demise of a biological system.

Figure 2

\section{Elementary network attributes in complex adaptive systems}

Common operational features illustrated in a network with hypothetical topology. Nodes (circles of different size) indicate system components with relative importance (e.g., red filled circles correspond to hubs with high connectivity), and links (lines with various thickness and arrows) indicate connection strength and directionality between pairs of nodes. Autoregulatory loops allow rapid initial response. Feedback allows response 
monitoring and adjustment. Feed-forward loops act as delay elements, sensitivity filters, pulse generators, or response accelerators. Single-input modules permit sequential activation and time-based expression. Multi-functional pathways share the same effector ('bow-tie'). Redundancy indicates the existence of parallel effectors resulting in a common outcome. Degeneracy is a special form of redundancy in which separate pathways yield the same outcome but may produce additional effects. Modules represent clusters of highly connected nodes specialized for a subtask. Network diameter is the longest distance between two nodes through the shortest possible route (path connecting green nodes between extremes marked with asterisk).

\section{Figure 3}

\section{Physiological adaptation: An integrated view}

A. Probability distribution of biological systems parameters depicted in the phase space remains narrow and predictable in health. Perturbations may cause pathophysiological conditions that prompt chaotic search for new attractors. Settling in these attractors may be temporary as ongoing perturbations escalate this explorative phase of adaptation increasingly associated with tradeoffs and compromises (sequelae-pathology). The system moves from intermediate pathophenotypes to disease pathophenotypes and beyond, corresponding to successive adaptation strategies (i.e., restore, explore, and abandon). B. Physiological adaptation in obesity is primarily determined by sustained nutrient excess, which may lead to intermediate pathophenotypes such as inflammation and altered energy metabolism (dashed circles). If perturbations persist, adaptation efforts to find new attractors are increasingly fraught by compromises and tradeoffs, resulting in higher risk for developing disease pathophenotypes marked by endothelial dysfunction (cardiovascular disease), insulin resistance (type 2 diabetes), and oncogenesis (cancer). C. Schematized network of attractors associated with obesity 
including links between relevant disease pathophenotypes and sub-phenotypes to illustrate hypothetical transition probabilities in the phase space recording a single life. Similar and more complex topologies could represent attractors that a biological system may occupy through physiological adaptation with opportunities and liabilities determined by the genetic and environmental assemblage of an individual organism (e.g., person-specific patterns of diabetic end-organ damage or cancer development). Computational analysis of personalized attractor networks may eventually prove invaluable in predicting and managing the outcomes of complex diseases. 
Figure 1

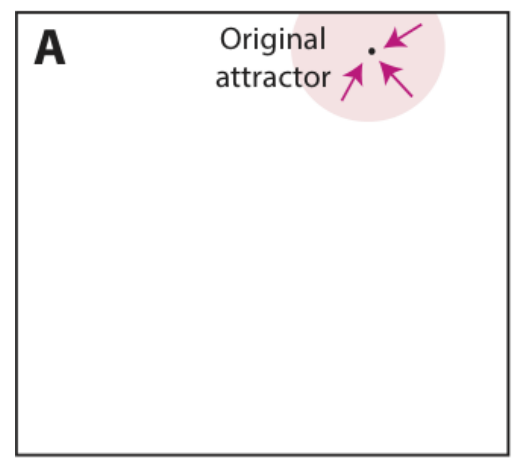

Ordered transitions:'restore'

(e.g., homeostasis)

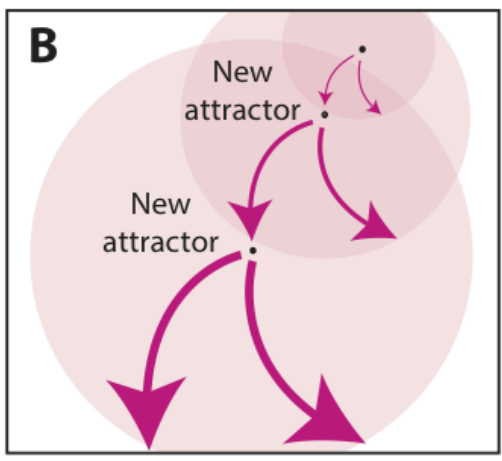

Chaotic search:'explore'

(e.g., allostasis)

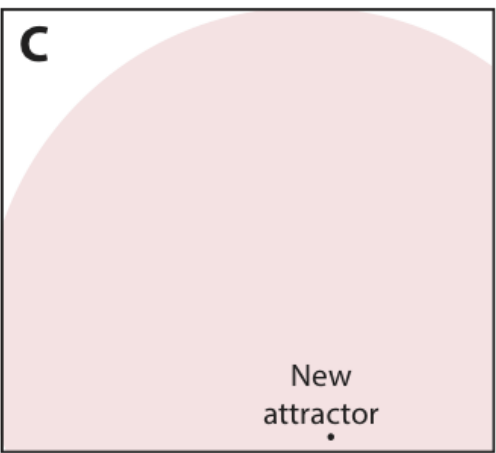

Static behavior: 'abandon' (e.g., allostatic crash) 
Figure 2

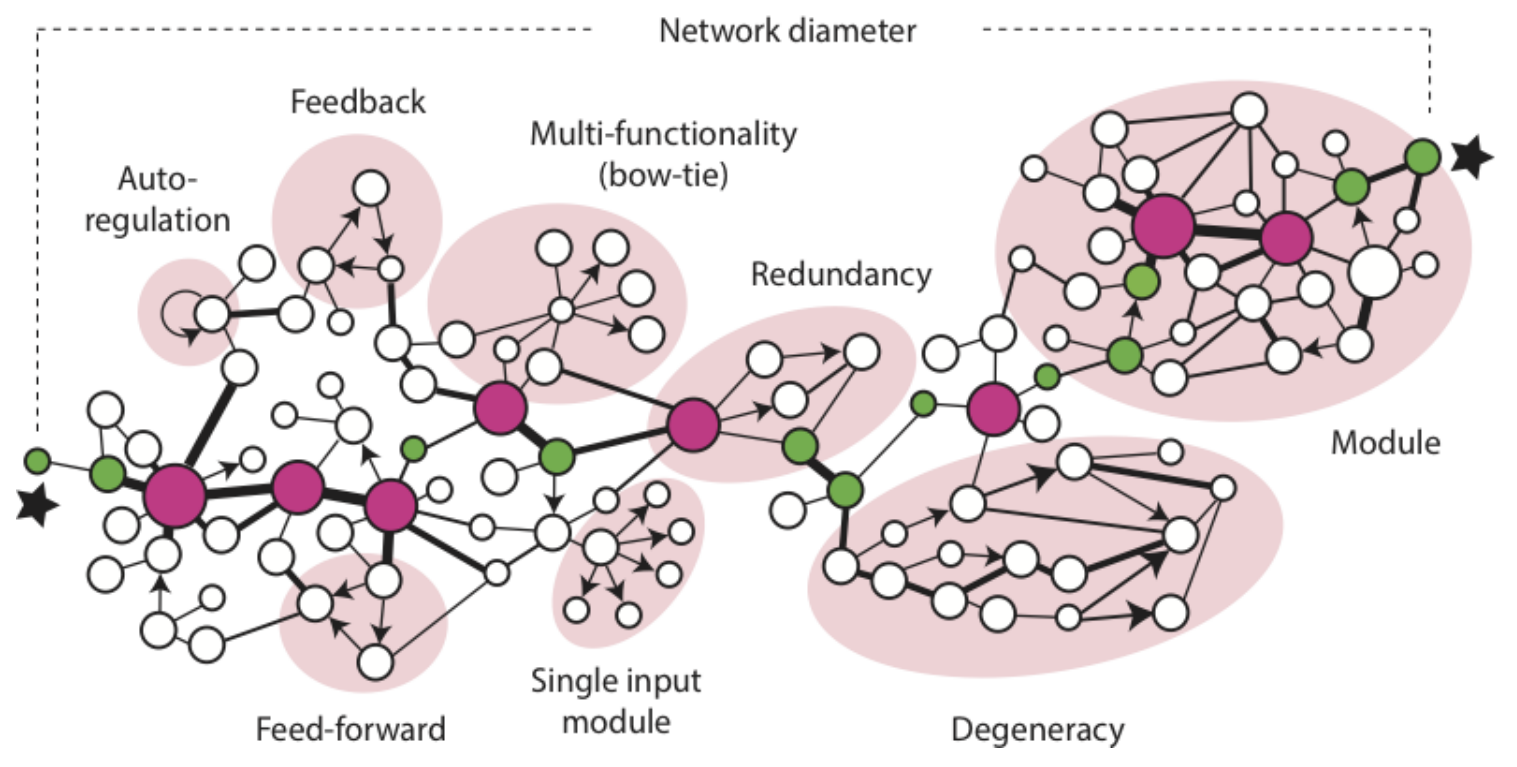


Figure 3
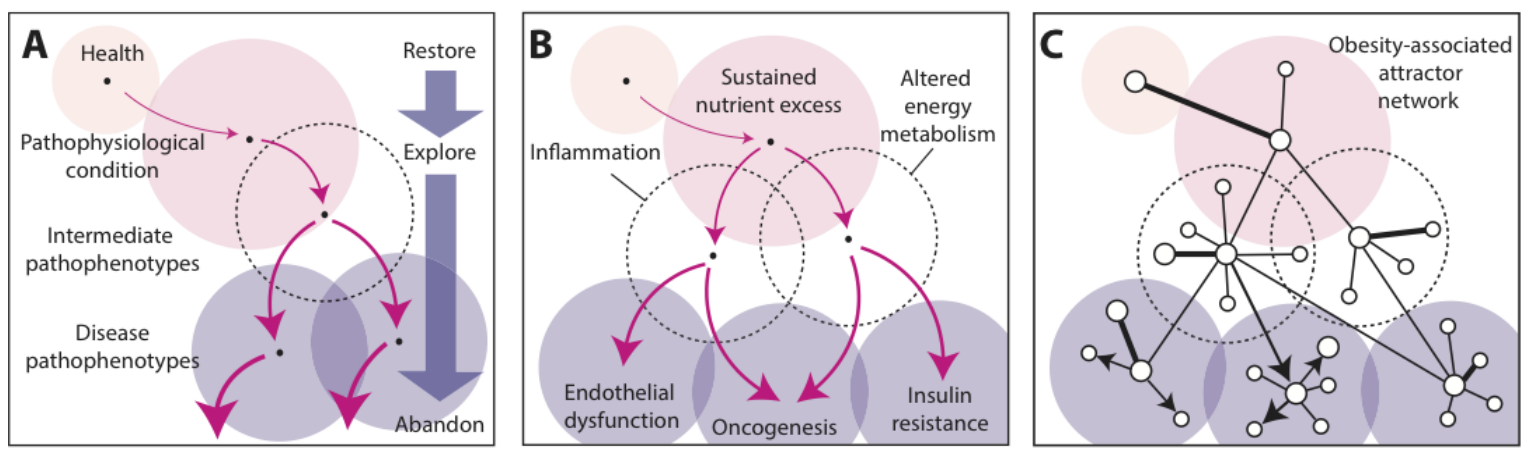